\title{
Finite-difference time-domain method calculation of light propagation through H-PDLC
}

\author{
V. Kubytskyi, V. Reshetnyak \\ Taras Shevchenko Kyiv National University, Physics Department \\ 2, prospect Academician Glushkov, 03680 Kyiv, Ukraine \\ E-mail: kubba@univ.kiev.ua
}

\begin{abstract}
Presented is the study of the diffraction properties of transmission holographic polymer dispersed liquid crystal (H-PDLC) grating. We constructed a two-dimensional model of H-PDLC film with cylindrical LC droplets. Director distribution inside LC droplets was calculated using the Monte-Carlo method. To calculate light propagation through the film we have solved numerically the Maxwell equations using the finitedifference time-domain method (FDTD). The FDTD analysis of diffraction was performed for $s$ - and $p$-polarized incident light. Externally applied electric field influence on the diffraction efficiency was studied.
\end{abstract}

Keywords: polymer dispersed liquid crystal, Monte-Carlo simulation, electric switch, finite-difference time-domain method.

Manuscript received 21.11.06; accepted for publication 26.03.07; published online 01.06.07.

\section{Introduction}

Holographic polymer dispersed liquid crystals (HPDLCs) are of recent interest due to a number of applications thereof include reflecting flat-panel displays, optical interconnects, diffraction lenses with switchable focus, optical data storage, application specific lenses, and image capture devices. H-PDLC materials are formed by exposing a light-sensitive homogeneous monomer and LC mixture by an interference pattern. The number of monomers is reduced in the stronger irradiated regions with the polymerization and the monomers from the dark regions diffuse into the illuminated zones. As a result of the above process, we get a grating made by a periodic distribution of small LC droplets embedded in a polymeric solid matrix. The main optical properties stemming from this periodic distribution can be easily controlled by an externally applied electric field. The high diffraction efficiency, high angular selectivity, low driving voltages, and fast switching times are the general requirements for H-PDLC transmission gratings. Many research groups make their efforts to improve these characteristics. A number of groups reported on the theoretical investigation of diffractive, polarization and switching properties of H-PDLCs. Sutherland et al. [1] developed a shaped-droplet model based on Montemezzani's coupled waves theory for the Bragg gratings [2] and Wu's model of droplet axis reorientation [3] to explain the switching curves of H-PDLC gratings. The effective medium theory and $2 \times 2$ matrix method were used by Sutherland [4] to model the Bragg diffraction and random scattering in reflective H-PDLC. In our previous work [5], we developed the model that combines Montemezzani's anisotropic coupled waves theory, Monte-Carlo simulations for the director profile within a droplet and statistical averaging with the orientational distribution function for droplet symmetry axes.

Here we present the results of direct calculation of light propagation through the H-PDLC.

\section{Model description}

H-PDLC is a complex optical structure. For theoretical investigation of light diffraction in this structure, the coupled waves theory for thick anisotropic hologram is often used. It was introduced by Montemezzani. The coupled waves theory gives approximate results. It considers only incident and diffracted to the first order waves, other orders of diffraction are neglected. The second-order derivatives of the wave amplitude are neglected in the slowly varying amplitude approximation.

We will use the FDTD calculation [6] of light propagation through the H-PDLC film by numerical solution of the Maxwell equations. The FDTD method provides a direct solution of the Maxwell equations by 
direct discretization of them considering both the spatial and temporal variations of fields. The equations are solved in a leap-frog manner. The electric field is solved at one point in time, then the magnetic field is solved at the next point in time, and the procedure is repeated. The FDTD method accommodate multidimensional inhomogeneity of the dielectric tensor, it is capable of directing spherical waves, plane waves or Gaussian beams while in arbitrary incidence on material. Now it is well developed to study the light propagation in isotropic and anisotropic media and its efficiency has been proved in various light-LC problems [16-18]. This consolidates us in the choice of such a tool. The success of the FDTD method depends on a special grid arrangement, where electric and magnetic field components are located in such a way that the first two Maxwell equations (sometimes known as the Gauss laws) are implicitly enforced, and the electric and magnetic fields are automatically divergence-free. This arrangement, known as the Yee cell [7] is the main distinctive feature of the method. Kane Yee disctretized the system of the Maxwell differential equations and expressed it as a set of finite differences.

The Yee discretization scheme in 2D case for anisotropic media is splitted into two systems of equations:

$$
\begin{aligned}
& E_{x}^{n+1}=E_{x}^{n}+\Delta t\left[\varepsilon_{x x}^{-1}\left(\frac{\partial H_{z}^{n+\frac{1}{2}}}{\partial y}\right)+\varepsilon_{x y}^{-1}\left(\frac{\partial H_{z}^{n+\frac{1}{2}}}{\partial x}\right)\right], \\
& E_{y}^{n+1}=E_{y}^{n}+\Delta t\left[\varepsilon_{y x}^{-1}\left(\frac{\partial H_{z}^{n+\frac{1}{2}}}{\partial y}\right)+\varepsilon_{y y}^{-1}\left(\frac{\partial H_{z}^{n+\frac{1}{2}}}{\partial x}\right)\right], \\
& H_{z}^{n+\frac{1}{2}}=H_{z}^{n-\frac{1}{2}}+\frac{\Delta t}{\mu}\left(\frac{\partial E_{x}^{n}}{\partial y}-\frac{\partial E_{y}^{n}}{\partial x}\right)
\end{aligned}
$$

for $\mathrm{TM}_{z}$ wave, and

$$
\begin{aligned}
& E_{z}^{n+1}=E_{z}^{n}+\Delta t\left[\varepsilon_{z z}^{-1}\left(\frac{\partial H_{y}^{n+\frac{1}{2}}}{\partial x}-\frac{\partial H_{x}^{n+\frac{1}{2}}}{\partial y}\right)\right], \\
& H_{x}^{n+\frac{1}{2}}=H_{x}^{n-\frac{1}{2}}+\frac{\Delta t}{\mu}\left(\frac{\partial E_{z}^{n}}{\partial y}\right), \\
& H_{y}^{n+\frac{1}{2}}=H_{y}^{n-\frac{1}{2}}+\frac{\Delta t}{\mu}\left(\frac{\partial E_{z}^{n}}{\partial x}\right)
\end{aligned}
$$

for $\mathrm{TE}_{z}$ wave, where $\left(E_{x}^{n+1}, E_{y}^{n+1}, E_{z}^{n+1}\right)$ and $\left(H_{x}^{n+\frac{1}{2}}, H_{y}^{n+\frac{1}{2}}, H_{z}^{n+\frac{1}{2}}\right)$ are the components of the electric and magnetic fields at $(n+1)$ and $\left(n+\frac{1}{2}\right)$ points of time, respectively. These two systems of equations represent two independent wave polarizations: $s$ polarized $\left(\mathrm{TE}_{\mathrm{z}}\right)$ and $p$-polarized $\left(\mathrm{TM}_{\mathrm{z}}\right)$ waves.

A scanning electron microscopy study shows that LC droplets form as nanoscale domains in H-PDLC gratings [8]. To simplify our model, we suppose that the LC droplets is cylindrical in 2D geometry. The dielectric permittivity tensor of LC is associated with a director distribution inside a droplet, and can be written as:

$\varepsilon_{i j}=\varepsilon_{\perp} \delta_{i j}+\varepsilon_{\alpha} n_{i} n_{j}$

where $\varepsilon_{a}=\varepsilon_{\|}-\varepsilon_{\perp} ; \quad n_{i}, \quad n_{j}$ are the director field components.

For further calculations, we suppose that the LC director projection on $z$ axes is equal to zero. In this case,

$\varepsilon_{x x}^{-1}=\frac{n_{y}^{2}}{\varepsilon_{\perp}}+\frac{1-n_{y}^{2}}{\varepsilon_{\|}}, \varepsilon_{y y}^{-1}=\frac{n_{x}^{2}}{\varepsilon_{\perp}}+\frac{1-n_{x}^{2}}{\varepsilon_{\|}}, \varepsilon_{z z}^{-1}=\frac{1}{\varepsilon_{\perp}}$.

We numerically simulate the director distribution inside the LC droplet to find the relative dielectric permittivity tensor of LC. The model that we used is the simplest model of nematic LC, which describes orientational interaction of molecules, this is the Lebwohl-Lasher lattice model [10], where the "particles" are treated as interaction sites ("spins") with continuously varying orientation but with fixed positions. The term particle refers to a local group of LC molecules with the same orientation. Minimization of free energy was realized using the Monte-Carlo method according to the Metropolis algorithm. We described it in our previous papers [5]. There are a few other models that describe properties of LC more realistically, but they are much more complicated.

With the size of LC droplets in the transmission $\mathrm{H}$ PDLC that is $50 \ldots 100 \mathrm{~nm}$, it comes necessary to use a fine grid for calculation of light propagation through the LC droplets. Fine discretization in specific region of computational space is often required to achieve the desired results. It saves computational resources as compared to simple decreasing of Yee grid step.

In Fig. 1, we show schematically the computational domain. For introduction of incident plane wave source into the FDTD lattice, we use the total field and the scatter field formulation [6]. The plane wave generates at the virtual interface between the total field and scatter field regions. It is based on a linearity of the Maxwell equations. Computational resources of computers are limited, because of this it is impossible to extend computational grid to infinity. Use of absorbing layers is the way to solve this problem. Computational domain is limited to Berenger perfectly matched layer (PML) [11]. This technique uses a special nonphysical lossy medium to absorb electromagnetic waves. A thin layer of this medium will absorb outgoing waves without reflection into computational domain. Calculation of field components Eqs (4)-(6) for each next moment of time results in propagation of the electromagnetic wave. The 


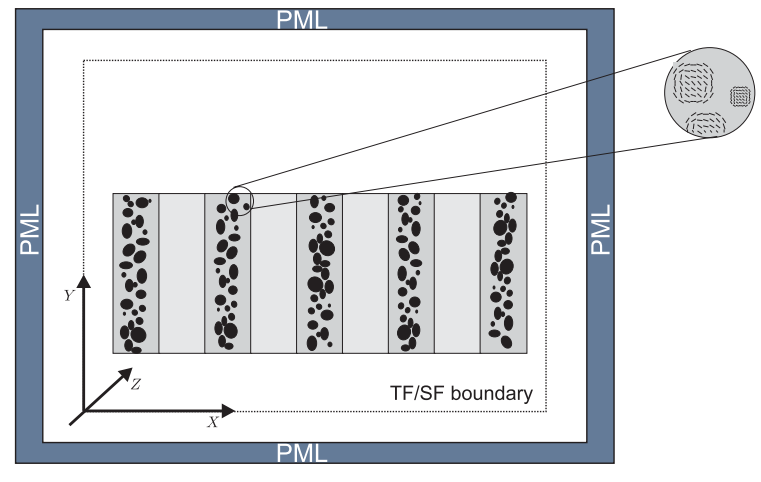

Fig. 1. Geometry of the computational domain.

near field obtained in simulation then transformed to far field using the electromagnetic equivalence principle. By this principle, it is necessary to calculate electric and magnetic currents in phasor domain and then project these currents to the required distance from the scatterer. Titus [9] in his dissertation simplified the Kirhhoff surface integral for the 1-D case:

$$
\begin{aligned}
& \psi^{\mathrm{far}}\left(x_{\mathrm{far}}, y_{\text {far }}\right)=\frac{e^{-i \pi / 4}}{\sqrt{8 \pi k}} \int_{-d}^{d} \frac{e^{-i k R}}{\sqrt{R}}\left[\frac{\partial}{\partial y} \psi^{\text {near }}\left(x^{\prime}, y_{0}\right)+\right. \\
& \left.+\frac{i k\left(y_{\mathrm{far}}-y_{0}\right) \psi^{\text {near }}\left(x^{\prime}, y_{0}\right)}{R}\right] d x^{\prime},
\end{aligned}
$$

where $R+\left\lfloor\left(x^{\prime}-x_{\text {far }}\right)^{2}+\left(y_{0}-y_{\text {far }}\right)^{2}\right\rfloor^{1 / 2}$ is the distance to the observation point $\left(x_{\text {far }}, y_{\text {far }}\right), k=2 \pi / \lambda$ is the incident wave vector, $\psi^{\text {near }}$ is the near field electric or magnetic component. Thus, the algorithm for solving the light propagation problem is as follows: we calculate the director profile inside the LC droplets by the MonteCarlo method; then we use the FDTD simulation to obtain a near-field diffraction pattern; the final step is the transformation of near-field data to far-field ones.

\section{Results}

We investigate the diffraction properties of hologram under the applied voltage for both $p$ - and $s$-polarized incident beams. We used the following parameters for simulation of H-PDLC film [1]: the thickness $d=8 \mu \mathrm{m}$, the grating period $\Lambda=1 \mu \mathrm{m}$, the width $L=40 \mu \mathrm{m}$, the dielectric permittivities $\varepsilon_{\perp}^{\mathrm{LC}}=2.356225, \varepsilon_{\|}^{\mathrm{LC}}=2.8224$, $\varepsilon_{\mathrm{pol}}=2.3409$, the incident light wavelength $\lambda=$ $0.633 \mu \mathrm{m}$.

For accurate simulation of H-PDLC, we need to set dielectric permittivities for every grid point. From the beginning, we set the dielectric permittivity for a rectangle with dimensions $d \times L$ equal to polymer dielectric permittivity. Then, we fill stripes inside this rectangle with LC droplets in such a way as to form the grating with the period $\Lambda$. The simulation program is constructed in such a manner that each LC droplet has corresponding class that specifies the dielectric permittivity tensor of the droplet. This class makes fine discretization of computational grid with refinement factor of 5 [12]. Thus, the grating period $\Lambda$ consist of the region with $\mathrm{LC}$ droplets of the width $\alpha \Lambda$ and polymer region with the width $(1-\alpha) \Lambda$. The volume fraction of LC in the stripe is $f_{c}$. Our model of H-PDLC is constructed according to the experimental works on investigation of the structure of $\mathrm{H}$ PDLC [14]. We study the diffraction properties of $\mathrm{H}$ PDLC for the angle of incidence equal to the Bragg one, which can be obtained from

$\theta_{\mathrm{B}}=\arcsin \left(\frac{\lambda_{0}}{2 n_{0} \Lambda}\right)$.

Here $\lambda_{0}$ is the incident light wavelength in vacuum, $n_{0}$ is the average refraction index of the grating.

To investigate the diffraction of $p$-polarized light, we used the system of Eqs (1)-(3) and Eq. (8) for the dielectric permittivity of the LC droplet. We set the initial orientation of axes of LC droplets along the grating vector. Effect of external electric field on $\mathrm{H}$ PDLC is calculated using the Monte-Carlo simulation for director profile. The value $E$ is measured in dimensionless units. The critical field $E=1$ corresponds to the applied electric field value of about $20 \mathrm{~V} / \mu \mathrm{m}$.

We define the diffraction efficiency according to [13] as the ratio between the intensity of a chosen diffraction order and the sum of the intensities of all the other diffraction orders. In our case, only the nearby orders give important contributions, which means:

$\eta=\frac{I_{-1}}{I_{-1}+I_{0}}$,

where $I_{0}$ denotes the intensity of the forward diffracted beam and $I_{-1}$ is the intensity of the 1 st order diffracted beam. Such a definition was used for compensation of various optical losses.

Fig. 2 illustrates the diffraction of $p$-polarized light for various values of applied external field. Our results show that the maximum of diffraction efficiency corresponds to zero applied field. It can be explained by big difference in dielectric permittivities of liquid crystal and polymer. Increasing of voltage leads to reorientation of LC director inside droplets. Because of this dielectric permittivity of the droplets changes. Modulation of dielectric tensor became small and diffraction efficiency decreased.

Earlier in Eq. (8), we supposed that the LC director has zero projection on the axis $z$. From this it follows that the s-polarized incident light will not diffract due to a small difference in the dielectric permittivities of $\varepsilon_{\perp}^{\mathrm{LC}}$ and $\varepsilon^{\mathrm{pol}}$. In fact, the experiments show that the $s$ polarized light is diffracted. We simulate a situation when $n_{z} \approx 1$ to study diffraction of the s-polarized light on H-PDLC. 


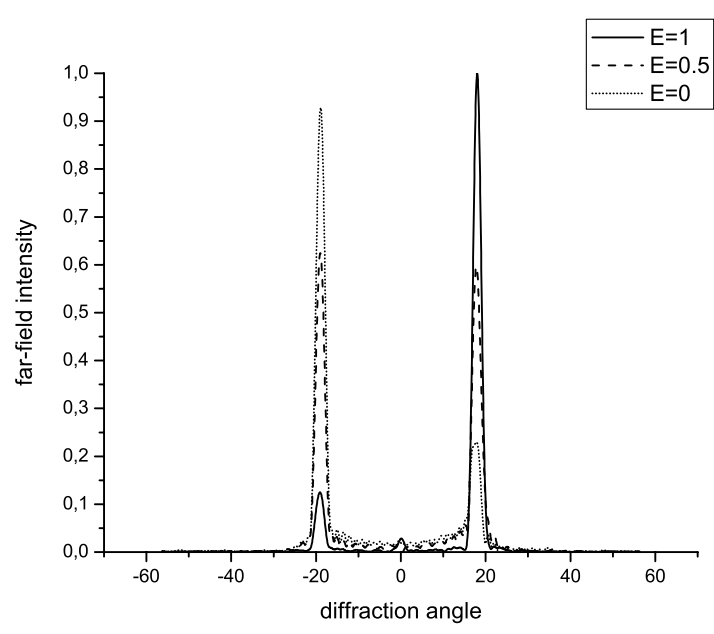

Fig. 2. Far-field intensity for diffraction of p-polarized light under external field. The $V$ value was measured in dimensionless units.

By the example of diffraction of the s-polarized light, we will discuss the difference between the approximate theory of Montemezzani and numerical simulation. The coupled waves theory for thick anisotropic holograms developed by Montemezzani and Zgonik assumes that the dielectric permittivity of media is periodically modulated as follows:

$\varepsilon=\varepsilon^{0}+\varepsilon^{1} \cos (K x)$,

where $\varepsilon^{0}$ is the average dielectric tensor of the composite and $\varepsilon^{1}$ is the amplitude of the dielectric tensor modulation. Sutherland [1] showed that, for H-PDLC grating, the dielectric permittivities can be expressed as:

$\varepsilon_{\|, \perp}^{0}=\varepsilon_{\|, \perp}^{\mathrm{LC}} \alpha f_{c}+\varepsilon^{\mathrm{pol}}\left(1-\alpha f_{c}\right)$,

$\varepsilon_{\|, \perp}^{1}=\frac{2 f_{c}}{\pi} \sin (\alpha \pi)\left(\varepsilon_{\|, \perp}^{\mathrm{LC}}-\varepsilon^{\mathrm{pol}}\right)$,

where $f$ is the bulk fraction of the dispersed material ( $f$ has the shape of a periodic rectangular wave that is zero in the solid polymer region and has a value $f_{c}$ in the PDLC region), the width of the PDLC region is $\alpha \Lambda$ (where $\alpha$ is a fraction of LC stripe in period), $\varepsilon_{\|}^{\mathrm{LC}}$ and $\varepsilon_{\perp}^{\mathrm{LC}}$ are the effective relative permittivities of $\mathrm{LC}$, and $\varepsilon^{\text {pol }}$ is the relative permittivity of the polymer. This approximation for effective dielectric constant holds as long as dielectric constants of the host and dispersed materials are nearly equal.

Fig. 3 shows diffraction of the s-polarized light on H-PDLC with smooth modulation of dielectric permittivity Eq. (12). The impinging plane wave has the

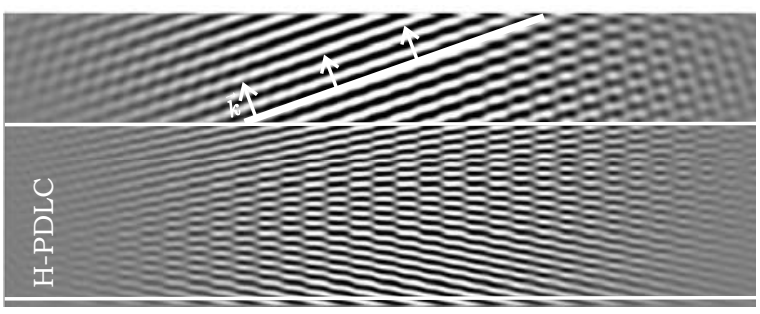

Fig. 3. Diffraction on H-PDLC with smooth modulation.

Gaussian profile to reduce the left and right computational domain boundaries effect on the calculation result. Amplitude of the electric field $E_{z}$ is showed by colors: dark grey is negative and light grey is positive. As shown in Fig. 3, light propagates through the H-PDLC and changes its phase so that at the output it forms the plane wave.

In Fig. 4, shown is diffraction on the H-PDLC with LC droplets. As clear from the figure, light diffracts similarly to the scheme in Fig. 3 with the difference that now we can see the scattering by the droplets.

Using the far-field diffraction pattern, we can compare the diffraction efficiencies of both gratings. Calculation of the diffraction efficiencies gives $\eta_{\text {smooth }}=0.83$ for the grating with smooth modulation of dielectric permittivity and $\eta_{\text {drops }}=0.75$ for that with LC droplets. Montemezzani showed that theoretically the diffraction efficiency of thick transmission holographic gratings can achieve $\eta=1$. We compare diffraction on H-PDLC grating composed of cylindrical LC droplets (where dielectric permittivity expressed as $\varepsilon_{z z}^{-1}=\frac{1}{\varepsilon_{\|}}$ (Fig. 4)) with that on grating with smooth modulation of dielectric permittivity Eq. (12) (Fig. 3). All parameters of the gratings are chosen to be the same. Figs 3-5 shows that the grating operates in the Bragg regime: light is incident angularly $18^{\circ}$ and diffracts to $-18^{\circ}$. The diffracted peaks are different because of light scattering by the LC droplets. We suppose that noise in Fig. 5 induced by scattering by the LC droplets.

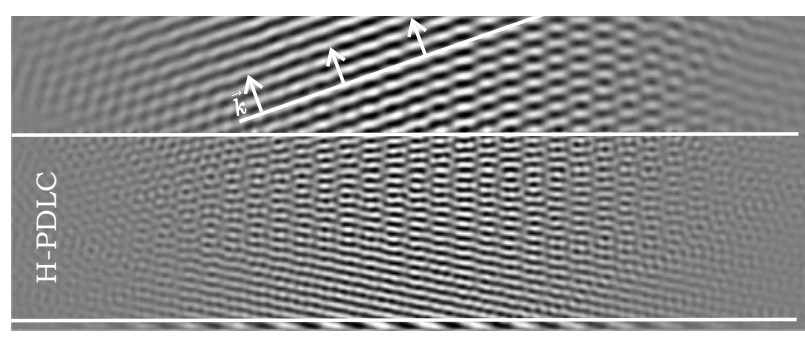

Fig. 4. Diffraction on H-PDLC composed of LC droplets. 


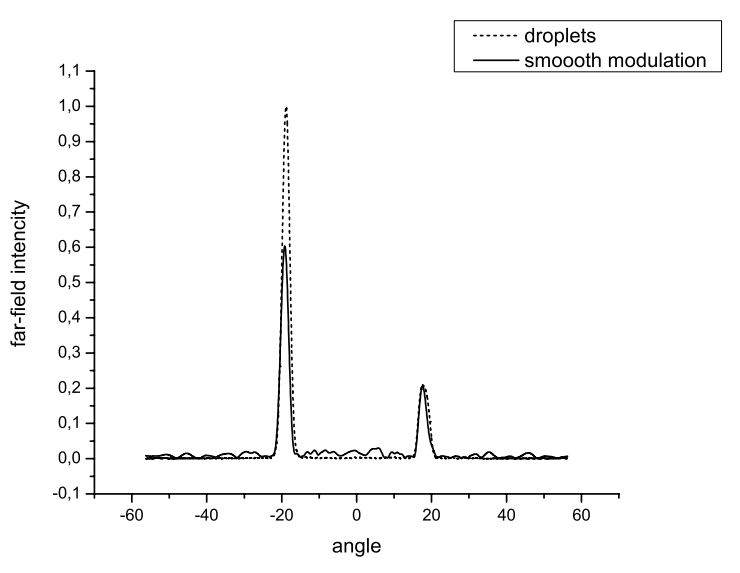

Fig. 5. Far-field intensity for H-PDLC with smooth modulation and H-PDLC composed of LC droplets. Diffraction of the spolarized light.

\section{Conclusions}

We have presented the model of H-PDLC transmission grating that combines the Monte-Carlo simulation for director profile inside cylindrical droplets and the FDTD calculation of light propagation through the film. It was shown that the grating operates in the Bragg regime. The developed model is suitable for computing the diffraction characteristics of holographic gratings.

We show the operating modes of the grating for $p$ polarized beam: diffraction mode - for the zero external field; intermediate mode - for voltages lower than critical; and transmission one - for electric field higher than critical. External field reorients the director inside LC droplets, what leads to changes in modulation dielectric tensor. This allows balancing between intensities of diffracted and transmitted beams and controlling the diffraction efficiency.

Comparison of diffraction of the s-polarized light on the H-PDLC (where stripes are composed of LC droplets) with H-PDLC with smooth modulation of dielectric permittivity (usually used in model of $\mathrm{H}$ PDLC) shows that there are "scattering" by the droplets. Due to this fact the intensity of diffracted beam for HPDLC with LC droplets is lower.

\section{Acknowledgements}

This work has been partially supported by: INTAS Young Scientist Fellowship Award 1000019-6375 to V. Kubytskyi (2007-8). NATO Grant CBP.NUKR.CLG. 981968 "Electro-optics of heterogeneous liquid crystal systems" awarded to V.Reshetnyak (2006-8); We also gratefully acknowledge discussions with Dr. Tigran Galstian.

\section{References}

1. 1. R.L. Sutherland // J. Opt. Soc. Amer. B 19, No.12, p. 2995-3003 (2002).

2. G. Montemezzani, and M. Zgonik // Phys. Rev. E 55, p. 1035-1047 (1997).

3. B.-G. Wu, J.H. Erdmann, and J.W. Doanne // Liquid Cryst. 5, p. 1453 (1989).

4. R.L. Sutherland, V.P. Tondiglia, L.V. Natarajan, P.F. Lloyd, and T.J. Bunning // J. Appl. Phys. 99, 123104 (2006)

5. V. Kubytskyi, V. Reshetnyak, T. Galstian // MCLC 438, p. 283-290 (2005).

6. A. Taflove, Computational electrodynamics: the finite difference time domain method, second edition. Artech House, 2000.

7. K. Yee // IEEE Trans. Antennas Propag. 14, p. 302-307 (1966).

8. R.T. Pogue, L.V. Natarajan, V.P. Tondiglia, S.A. Siwecki, R.L. Sutherland, and T.J. Bunning // Proc. SPIE 3475, p. 2-11 (1998).

9. C.M. Titus, Refractive and diffractive liquid crystal beam steering devices. Ph.D. dissertation in Kent State University, 2000.

10. P.A. Lebwohl and G. Lasher // Phys. Rev. A 6, p. $426(1972)$

11. J.-P. Berenger // J. Comp. Phys.114, p. 185-200 (1994).

12. M.W. Chevalier, R.J. Luebbers, and V.P. Cable // IEEE Trans. Antennas Propagat. 45, p.411-421 (1997).

13. I. Drevensek-Olenik, M. Fally, M.A. Ellabban // Phys. Rev. E 74, 021707 (2006).

14. T.J. Bunning, Holographic polymer-dispersed liquid crystals (H-PDLCs) // Annu. Rev. Mater. Sci. 30, p. 83-115 (2000).

15. H. Kogelnik // Bell Syst. Tech. J. 48, p. 2909-2947 (1969).

16. B. Witzigmann, P. Regli, W. Fichtner // J. Opt. Soc. Amer. A 15, p. 753 (1998).

17. D.K. Hwang, A.D. Rey // Liquid Cryst. 32, p. 483 (2005).

18. V. Ilyina, S.J. Cox, T.J. Sluckin // Opt. Communs 260, p. 474-480 (2006). 